\title{
Auto-Sintonia de Controladores PID Utilizando Logica Fuzzy Tipo-2 Intervalar para as Margens de Fase e de Ganho do Sistema.
}

\author{
José B. do C. Neto* Otacílio da M. Almeida** \\ Francisco V. E. Lemos ** Gabryel F. Soares* \\ Luno G. de Oliveira ${ }^{* *}$ José Maria P. Menezes Jr ${ }^{* *}$ \\ * Departamento de Engenharia Elétrica, Universidade Federal do \\ Piauí, PI, (e-mail: jose.borges90@hotmail.com), (e-mail: \\ gabryelfig18@gmail.com) \\ ** Programa de Pós-Graduação, Universidade Federal do Piauí, PI, \\ (e-mail: otacilio@ufpi.edu.br),(e-mail:victorleteves@gmail.com), \\ (e-mail: lunoeste@gmail.com), (e-mail: josemenezesjr@gmail.com)
}

\begin{abstract}
In this paper we propose a new algorithm for auto tuning of PID controllers whose gains are calculated through a self-tuning of the phase and gain margins for the closedloop system using Interval Fuzzy Type-2 Logic. Simulations and practical applications to real processes are presented with the objective of verifying the proposed control method.

Resumo: Neste artigo propõe-se um novo algoritmo para auto sintonia de controladores PID, em que os ganhos são calculados através de um auto ajuste das margens de fase e de ganho para o sistema em malha fechada, utilizando Lógica Fuzzy tipo-2 Intervalar. Simulações e aplicações práticas a processos reais são apresentados com o objetivo de verificar o método de controle proposto.
\end{abstract}

Keywords: PID control, Interval Type-2 Fuzzy, Fuzzy control, Phase Margin, Gain Margin .

Palavras-chaves: Controle PID; Fuzzy tipo-2 Intervalar; Controle Fuzzy; Margem de Fase, Margem de Ganho.

\section{INTRODUÇÃO}

O controlador PID é atualmente a estrutura de controle mais empregada em sistemas industriais. Tal fato decorre de sua rapidez de atuação, simplicidade e eficiência no controle de processos (Berner et al., 2018). Entretanto, ele é um controlador considerado convencional, ou clássico, e no projeto de tais controladores, considera-se apenas as relações lineares entre as variáveis do processo controlado, o que impõe severas limitações no desempenho de controle, frente a sistemas que possuem modelagem complexas, nãolinearidades ou incertezas (Åström and Hägglund, 2001).

Para superar as desvantagens que os controles convencionais apresentam, foram desenvolvidas técnicas de Inteligência Computacional Aplicadas (ICAs) para o controle de processos (Chaiyatham and Ngamroo, 2017; Dai et al., 2017; Santana et al., 2018; Coimbra et al., 2017). Uma das aplicações de ICAs são os Controladores PID Inteligentes (Almeida and Coelho, 2002). O desenvolvimento destas técnicas e algoritmos de inteligência computacional, aliados ao desenvolvimento de hardware dos últimos anos, tornou possível o desenvolvimento de sistemas de controle automáticos, que exibem comportamentos inteligentes, como solução para suprir deficiências dos sistemas de controle convencional (Kruse et al., 2016).
Entre estes controladores destacam-se aqueles baseados na computação nebulosa (Fuzzy). Nos quais consistem em conjunto de regras linguísticas que utilizam a teoria dos conjuntos nebulosos para transformar um conjunto de regras, em uma coerente estratégia de controle (Almeida and Coelho, 2002).

A idéia de utilizar controladores Fuzzy em processos industriais surgiu em 1974 com o trabalho de Mamdani (1974), já os primeiros trabalhos que abordavam a estruturas PID Fuzzy surgiram em 1990 com o trabalho de Kwok et al. (1990). Para aplicar a computação nebulosa ao controle convencional PID utiliza-se a estrutura de controle Gain Sheduling, que tem como princípio alterar os valores dos parâmetros de controle em função das variáveis conhecidas e monitoradas da dinâmica do sistema (Qiao et al., 2017). Desta forma, os parâmetros $K_{p}, K_{i}$ e $K_{d}$ são sintonizadas por uma base de regras e uma máquina de inferência Fuzzy, e o sinal de controle é gerado pela estrutura clássica do controlador PID (Chaiyatham and Ngamroo, 2017).

Para incorporar à lógica Fuzzy convencional do tipo 1 (Tipo-1) maior capacidade em lidar ou acomodar as incertezas linguísticas e numéricas em ambientes não estruturados (Hagras, 2007) foi introduzida por Zadeh (1975) o conceito da lógica Fuzzy tipo-2 (Tipo-2). Os conjuntos Fuzzy de tipo 2, são uma extensão dos Tipo-1 que adicio- 
nam um grau de liberdade extra, o que permite o sistema lidar melhor com a incertezas (Tai et al., 2016).

Sendo assim, este trabalho propõe uma nova forma de auto sintonia de controladores PID cujos os ganhos são calculados através de um auto ajuste das margens de fase e de ganho para o sistema em malha fechada, utilizando uma máquina de inferência Fuzzy tipo-2 Intervalar. Foram realizadas uma aplicações práticas a processos reais com o objetivo de verificar o método proposto.

O artigo está dividido como segue: na Seção 2, temos a descrição do controlador PID Fuzzy Baseado em Margem de Fase e de Ganho, na Seção 3, é abordado o controlador PID Fuzzy tipo-2 Intervalar baseado nas margens de fase e de ganho; já na Seção 4 são descritos os resultados das simulações realizadas, e das aplicações práticas; a Seção 5 encerra o artigo com as conclusões.

\section{ESTRUTURA DO CONTROLADOR PID FUZZY BASEADO EM MARGEM DE FASE E DE GANHO}

Zhao et al. (1993) apresentaram uma estrutura de controlador baseada em escalonamento on-line dos ganhos dos controlador PID utilizando lógica Fuzzy. Almeida and Coelho (2002) adotaram uma estrutura similar, mas que se baseou nos valores das margens de fase e de ganho do processo. No presente trabalho foi aplicado um método de escalonamento dos ganhos do controlador PID. Para isso uma máquina de inferência Fuzzy é utilizada para aferir os valores dos ganhos associados ao PID a cada período de amostragem do processo em tempo real. A estrutura do controlador é apresentada na Figura 1.

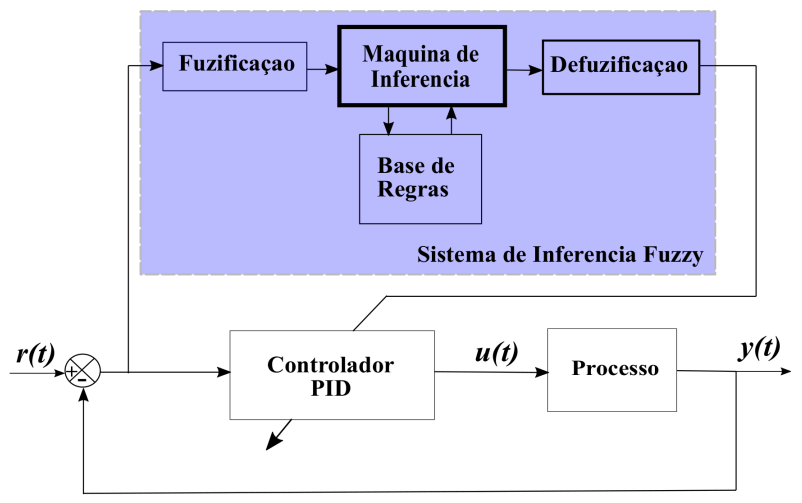

Figura 1. Estrutura do Controlador PID Fuzzy Baseado em Margem de Fase e de Ganho

O sistema proposto, assim como em qualquer controle convencional tem como objetivo minimizar a diferença entre $r(t)$ e $y(t)$, o que é definido na literatura como o erro $(e(t))$ em malha fechada . Para isso a cada período de amostragem o sinal de controle $u(t)$ tem seu valor aferido pelo sistema de inferência Fuzzy (SIF) e aplicado a entrada do processo. Neste trabalho, o SIF adotado tem como entradas o erro, $e(t)$, e a variação de erro, $\Delta e(t)$ e a saída é o valor da margem de ganho do processo $A_{m}$ para a planta do motor CC.

\subsection{Sintonia do controlador PID}

Para que seja possível utilizar o esquema de controle Fuzzy baseado em margem de fase e de ganho é necessário que o controlador PID tenha seus parâmetros iniciais sintonizados. Para que assim a máquina de inferência Fuzzy possa ser projetada.

Neste trabalho esta sintonia foi feita utilizando o método de Åström and Hägglund (1984), baseado na identificação automática da planta pelo método do relé.

A margem de ganho do processo $A_{m}$ é a variável do processo que é aferida a cada período de amostragem. A variação de $A_{m}$ é definida entre um valor máximo, $A_{m, \max }$ e um valor mínimo, $A_{m, m i n}$, com base no conhecimento de especialistas na dinâmica do processo a ser controlado. A fim de facilitar o tratamento matemático do controlador os valores de $A_{m}$ são normalizados entre zero e um, de acordo com (1), onde $A_{m}^{\prime}$ é o valor da margem de ganho normalizada.

$$
A_{m}^{\prime}=\left(A_{m}-A_{m, \min }\right) /\left(A_{m, \max }-A_{m, \min }\right)
$$

O valor de $A_{m}^{\prime}$ é determinado a partir da máquina de inferência Fuzzy projetada.

\subsection{Máquina de Inferência Fuzzy}

Uma máquina de inferência Fuzzy é um mecanismo de tomada de decisão baseado nos conhecimentos de especialistas. É composta por regras que descrevem o comportamento que a planta deveria ter. As regras utilizadas são da seguinte forma:

$$
\text { Se e }(t) e ́ A_{i} \quad e \quad \Delta e(t) e ́ B_{i} \quad \text { Então } A_{m, i}^{\prime}(t) \text { é } C_{i}
$$

$A_{i}, B_{i}$ e $C_{i}$ são conjuntos Fuzzy, $A_{m, i}^{\prime}$ é o valor da margem de fase para a $i$-ésima regra . O mecanismo de inferência é ilustrado na Figura 2. As operações da máquina de inferência Fuzzy são realizadas tendo como base um conjunto de regras nebulosas, chamada base de regras. Para este trabalho a base de regras foi derivada da resposta ao degrau em malha aberta de um sistema de $2^{\circ}$ ordem, tendo como base o trabalho de (Almeida and Coelho, 2002). Os conjuntos Fuzzy foram definidos como N - Negativo, Z - Zero, P - Positivo, para as variáveis de entrada e Pe - Pequeno, Me - Médio, Gr - Grande, para as variáveis de saída. A base de regras utilizada é mostrada na Tabela 1.

Tabela 1. Base de Regras para o Controlador PID Fuzzy Baseado em Margem de Fase e de Ganho

\begin{tabular}{cccc}
\hline $\mathrm{E} / \Delta E$ & $\mathbf{N}$ & $\mathbf{Z}$ & $\mathbf{P}$ \\
\hline $\mathbf{N}$ & $\mathrm{Pe}$ & $\mathrm{Me}$ & $\mathrm{Pe}$ \\
$\mathbf{Z}$ & $\mathrm{Gr}$ & $\mathrm{Gr}$ & $\mathrm{Gr}$ \\
$\mathbf{P}$ & $\mathrm{Pe}$ & $\mathrm{Me}$ & $\mathrm{Pe}$ \\
\hline
\end{tabular}

O processo de defuzificação é realizado de acordo com 3 . Onde $\mu_{i}$ é o valor verdade da regra $i$, que é obtido a partir do produto dos valores das FP dos antecendentes da $i^{\mathrm{a}}$ regra.(Almeida and Coelho, 2002)

$$
A_{m}^{\prime}=\sum_{i=1}^{n} \mu_{i} A_{m, i}
$$




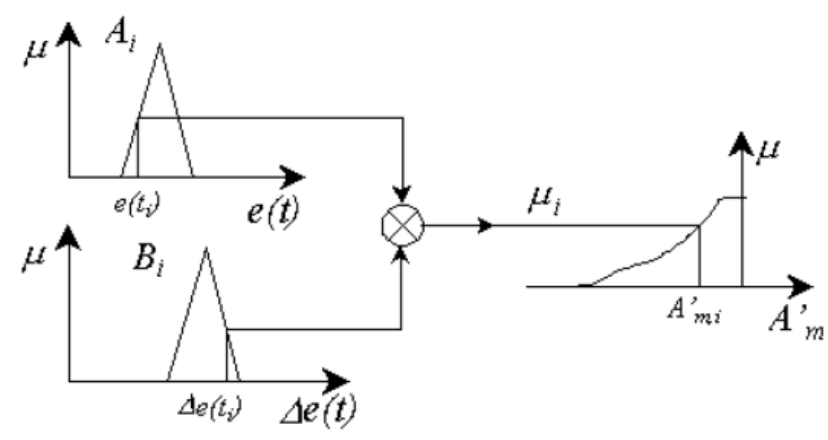

Figura 2. Processo de inferência de uma regra nebulosa

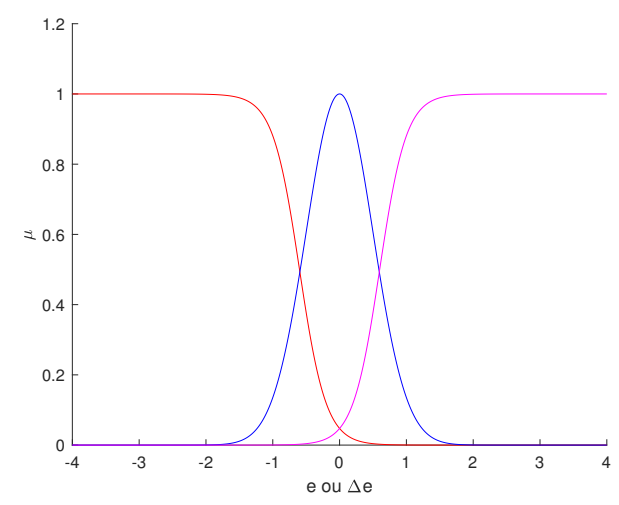

Figura 3. Funções de pertinência Sigmoide e Gausiana.

\subsection{Funções de pertinência}

Os conjuntos Fuzzy relacionam as variáveis numéricas com variáveis linguísticas. Um conjunto Fuzzy $A$ em $X$ é caracterizado por uma função de pertinência (FP) que associa cada ponto em $\mathrm{X}$ a um valor real entre 0 e 1 , que representa o valor de pertinência de x a A (Zadeh, 1965). Neste trabalho os conjuntos $\mathrm{N}$ e P são caracterizados por funções sigmoides da forma dada em 5 e 6 , já o conjunto $\mathrm{Z}$ é caracterizado por uma função gaussiana da forma apresentada em 4 .

$$
\begin{gathered}
\mu_{Z}(x)=\exp \left[\left(-\frac{1}{2}\right)\left(\frac{(x-m)}{\sigma}\right)^{2}\right] \\
\mu_{N}(x)=1-\left(1 /\left(1+e^{-a x+b}\right)\right) \\
\mu_{P}(x)=\left(1 /\left(1+e^{-a x+b}\right)\right)
\end{gathered}
$$

\subsection{Algoritmo Genético}

Para encontrar as funções de pertinência de saída foi utilizado um algoritmo genético para encontrar os valores ótimos para os valores dos conjuntos Fuzzy CRISP de saída $P e, M e$ e $G r$. Algoritmos genéticos (AG) tem como ideia básica manter uma população de cromossomos competindo através de processos de reprodução e mutação em um universo controlado a fim de encontrar o individuo que represente a melhor solução (Herrera et al., 1998). Cada cromossomo representa uma solução possível de um dado problema. A qualidade desta solução é mensurada

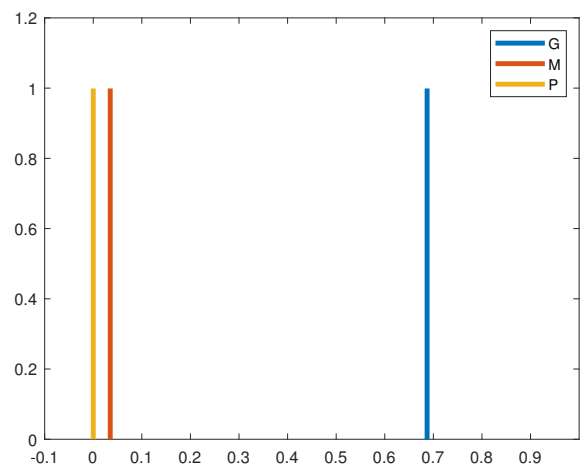

Figura 4. Funções de pertinência Singleton.

a partir de uma função de custo, que retorna um valor numérico para cada individuo. A partir destes valores os indivíduos são classificados, e com base nesta classificação são escolhidos os indivíduos para participar das fases de reprodução e mutação.

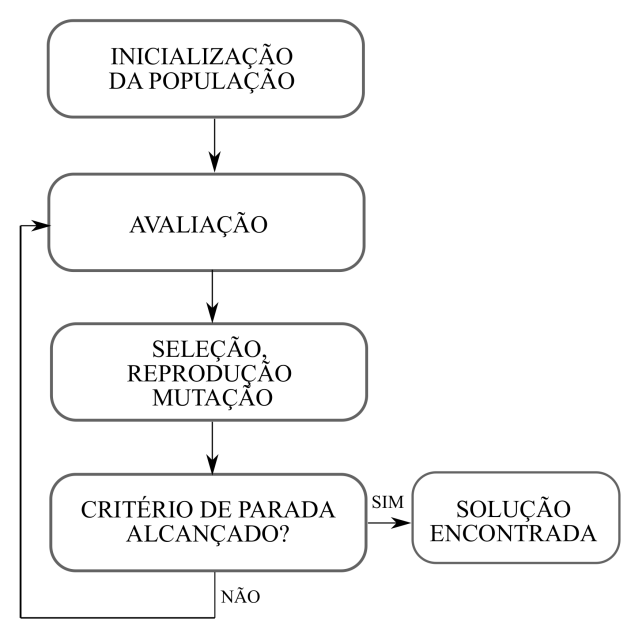

Figura 5. Estrutura de um algoritmo genético

Para encontrar os valores de $P e, M e, G r$ foi criado um AG com as seguintes características:

- Número de cromossomos: 64;

- Número de genes: 3;

- Número de gerações: 50;

- Taxa de reprodução: $85 \%$;

- Taxa de mutação: 15\%;

Os critérios de parada adotados foram a convergência da população ou o número máximo de gerações. A função objetivo adotada foi o índice de desempenho baseado no erro integral do tempo multiplicada pelo erro quadrático ou Integral of the time multiplied by square error (ITSE), definido por (7), onde T é o valor do horizonte de análise.

$$
I T S E=\int_{0}^{T} t e^{2} d t
$$

A escolha desta função objetivo se deve a sua recorrente aplicação em problemas de sintonia de controladores PID por técnicas meta-heurísticas. 
As funções de pertinência dos tipos gaussiana, sigmoide utilizadas estão ilustradas na Figura 3. As funções de pertinência do tipo Singleton, resultantes da otimização por AG para a saída, estão ilustradas na Figura 4.

\subsection{Sistema Fuzzy Tipo-1}

O sistema Fuzzy implementado tem as seguintes características:

- 2 variáveis de entrada: Erro e a taxa de variação do Erro;

- 1 variável de saída: o valor da margem de fase para o processo;

- Mecanismo de inferência do tipo Mamdani produtosoma, com 9 regras;

- A defuzzificação é feita pela média ponderada dos valores obtidos em cada uma das 9 regras.

\section{ESTRUTURA DO CONTROLADOR PID FUZZY TIPO-2 INTERVALAR BASEADO EM MARGEM DE FASE E DE GANHO}

O controlador PID Fuzzy tipo-2 Intervalar baseado em margens de fase e de ganho (PID-FT2I-FG) possui uma estrutura similar a do controlador PID Fuzzy baseado em margem de fase e de ganho, como mostrado na Figura 6 . Desta forma, toda a sintonia inicial do controlador PID, para este caso, foi realizada como descrita na Seção 2.

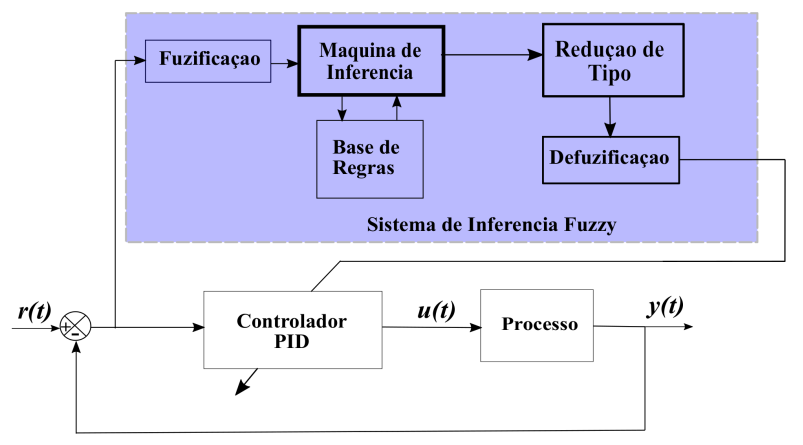

Figura 6. Estrutura do controlador Fuzzy tipo-2 Intervalar PID Baseado em Margem de Fase e de Ganho .

A diferença precípua entre Fuzzy Tipo-1 e Tipo-2 está associada precisamente à natureza da função de pertinência. Esta tem como principal característica incorporar uma área chamada de footprint of uncertainty (FOU), que representa a união de todas as funções de pertinência primárias (Karnik et al., 1999). Na Figura 7 tem-se a representação de um exemplo de FOU da função de pertinência gaussiana de tipo-1. A FOU é abordada como a área entre duas funções de pertinência que aproximam os limites das áreas que representam as incertezas associadas a variável linguística. Desta forma, representa-se uma FP do tipo-2 como duas FP do tipo-1, uma associada ao limite superior(FPS) e a outra ao limite inferior(FPI).

Segundo Wu and Tan (2004) existem duas formas de se desenvolver um sistema Fuzzy Tipo-2. A primeira é a abordagem parcialmente dependente, onde primeiramente o melhor sistema Fuzzy Tipo-1 é projetado, e em seguida

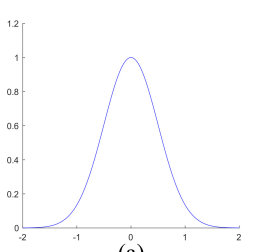

(a)

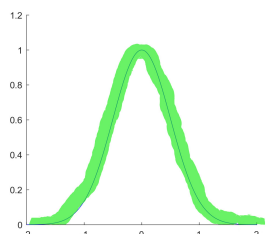

(b)

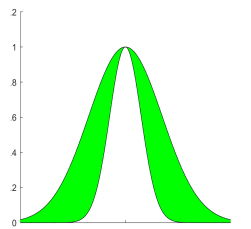

(c)
Figura 7. (a) Type-1 MF, (b) blurred type-1 MF (artistic rendition), and (c) footprint of uncertainty.

utilizado como parâmetros inicias do sistema Fuzzy Tipo2 , enquanto na segunda abordagem, o processo de desenvolvimento é totalmente independente, ou seja, os parâmetros são projetados diretamente para o sistema Fuzzy Tipo-2. Neste trabalho adota-se como ponto de partida o sistema Fuzzy Tipo-1 descrito na seção 2.

Para isso, as funções de pertinência da entrada foram transformadas em funções de pertinência do Tipo-2, e isso foi feito utilizado um algoritmo genético com codificação real para encontrar as melhores FPS e FPI para as curvas gaussianas e sigmoides utilizadas na entrada. Para as saídas foram adotadas funções de pertinência CRISP do Tipo-2, centradas nos valores definidos para o sistema Tipo-1.

Para encontrar as melhores FPS e FPI, utilizou-se uma FP do tipo 1 como base, e a partir dela foi feito o cálculo dos valores de $\sigma$ para as curvas gaussianas e de $b$ para as sigmoides para as FPS e FPI. Os cromossomos foram organizados como um vetor de tamanho 12 onde cada valor representa o desvio que aquela curva teria em relação a FP tipo 1 utilizada como base. Desta forma os valores de $\sigma$ e $b$ foram calculados a partir da soma dos valores de base com os valores do cromossomo mais apto obtido pelo algoritmo genético.

O AG de codificação real desenvolvido foi baseado no trabalho de Takahashi and Kita (2001) e possui as seguintes características:

- Número de cromossomos: 128;

- Número de genes: 12 ;

- Número de gerações: 50;

- Taxa de reprodução: $85 \%$;

- Taxa de mutação: $5 \%$;

- Fitnes Function: ITSE $=\int_{0}^{T} t e^{2} d t$.

Nas Figuras 8 e 9, são mostradas, respectivamente, as funções de pertinência das entradas, sendo elas o erro e a taxa de variação do erro, e as funções de pertinência de saída. A função em azul representa o conjunto Fuzzy N, a função em verde representa o conjunto Fuzzy $\mathrm{Z}$ e a função em amarelo representa o conjunto Fuzzy P.

\subsection{Sistema Fuzzy Tipo-2 Intervalar}

O sistema Fuzzy tipo-2 Intervalar implementado tem as seguintes características:

- 2 variáveis de entrada: Erro e a taxa de variação do erro;

- 1 variável de saída: o valor da margem de fase para o processo; 


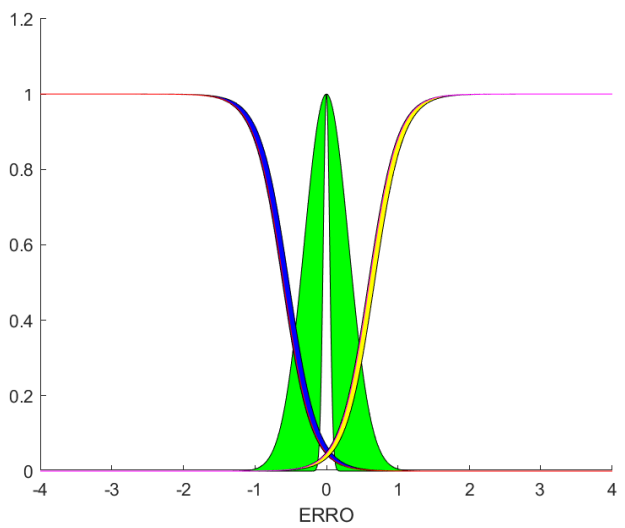

Figura 8. Funções de pertinencia otimizadas para o erro

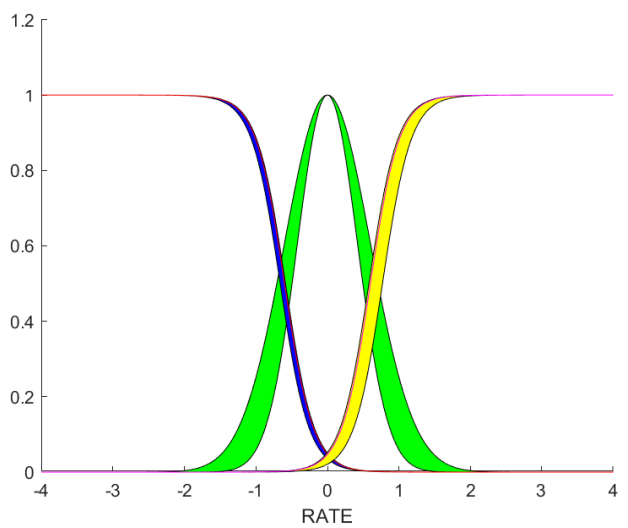

Figura 9. Funções de pertinência otimizadas para a variação do erro

- Mecanismo de inferência do tipo Mamdani produtosoma, com 9 regras;

- O algoritmo de redução de tipo implementado foi o EIASC, proposto por Wu and Nie (2011);

- A defuzificação é feita pela média dos valores obtidas na etapa de redução de tipo.

\section{RESULTADOS}

As simulações computacionais foram realizadas no software MATLAB. As simulações utilizaram um modelo do motor CC identificado pelo método dos mínimos quadrados não recursivo. Foram realizados ensaios em malha aberta com o motor $\mathrm{CC}$, nele foi aplicado sucessivos degraus com valores diferentes a fim de verificar seu comportamento dinâmico. Como resultado, obteve-se o ganho da planta alterando-se conforme os valores dos degraus de entrada são aumentados. Conferindo a ela um comportamento não-linear.

Para a experimentação pratica do controlador foi utilizada uma planta didática desenvolvida no Grupo de Pesquisa em Automação e Sistemas Inteligentes GRASI-UFPI. Ela é composta por um motor CC e uma placa microcontrolada utilizada para interfacear o sistema com o computador.

Na Tabela 2 apresenta os valores dos índices de desempenho utilizados na análise da qualidade dos algoritmos de controle proposto. Primeiramente são analisados o pa-

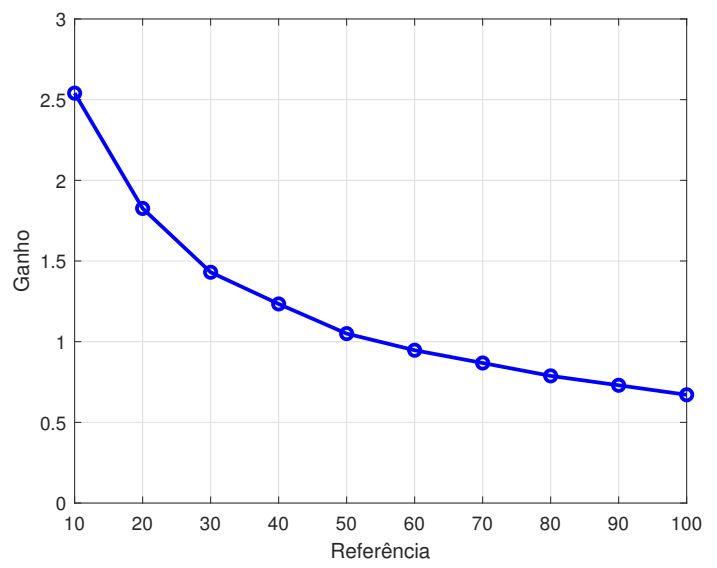

Figura 10. Resposta da planta ao degrau para valores de entrada diferentes

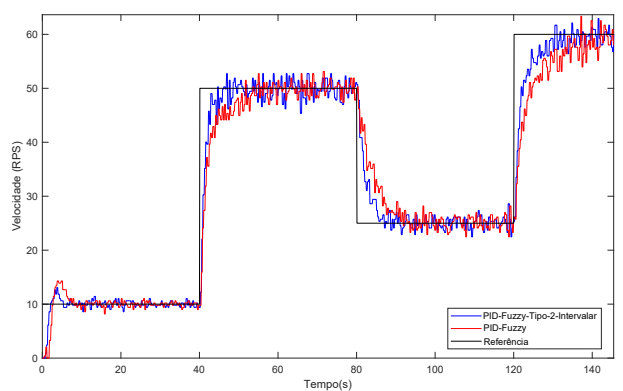

Figura 11. Velocidade controlada planta real

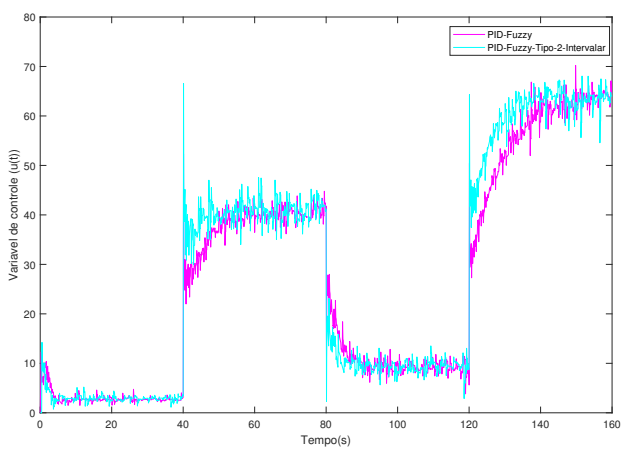

Figura 12. Sinal de controle planta real

râmetros baseados no erro, a Integral absoluta do erro ou Integral absolute error (IAE), a integral do erro quadratico ou Integral square error (ISE), integral do tempo multiplicada pelo erro absoluto ou Integral of the time multiplied by absolute error (ITAE), A integral do tempo multiplicada pelo erro quadrático ou Integral of the time multiplied by square error (ITSE), em seguida são analisadas as variâncias da variável controlada $s \alpha_{y}^{2}$ e da variável de controle $s \alpha_{u}^{2}$.

Os resultados obtidos a partir das simulações computacionais e da aplicação na planta real demonstram a melhoria introduzida na malha de controle quando foi utilizada a estrutura proposta. Houve uma redução no esforço de controle sobre a planta caracterizado pela diminuição dos 
Tabela 2. Resultados para o motor CC

\begin{tabular}{cccc} 
Índice & Fuzzy PID & Fuzzy PID Tipo 2 Intervalar & Melhoria \\
\hline IAE & $4.8926 \mathrm{e}+03$ & $3.4283 \mathrm{e}+03$ & $30.48 \%$ \\
ISE & $5.9006 \mathrm{e}+04$ & $3.8031 \mathrm{e}+04$ & $35.58 \%$ \\
ITAE & $4.1887 \mathrm{e}+05$ & $2.9264 \mathrm{e}+05$ & $30.13 \%$ \\
ITSE & $4.8971 \mathrm{e}+06$ & $3.0678 \mathrm{e}+06$ & $37.35 \%$ \\
I & $6.9453 \mathrm{e}+04$ & $5.4076 \mathrm{e}+04$ & $22.14 \%$ \\
$s \alpha_{y}^{2}$ & 43.3010 & 33.4242 & $22.80 \%$ \\
$s \alpha_{u}^{2}$ & 82.7173 & 35.0786 & $57.58 \%$ \\
\hline
\end{tabular}

índices $I, s \alpha_{u}^{2}$. O que mostra uma diminuição na agressividade da ação de controle, o que preserva a vida útil dos atuadores. Além disso, todos os índices de desempenho baseados em erro tiveram seus valores atenuados, o que demonstrar um melhoria global no desempenho do controlador.

\section{CONCLUSÕES}

Este trabalho teve como objetivo apresentar uma novo algoritmo de sintonia de controladores PID utilizando lógica Fuzzy tipo-2 Intervalar, baseada em margens de fase e de ganho. $\mathrm{O}$ algoritmo foi implementado com sucesso em linguagem MATLAB e seu desempenho foi aceitável para a aplicação realizada. Ele proporcionou uma melhora global do controle do processo.

Para trabalhos futuros, espera-se aperfeiçoar o algoritmo de sintonia das funções de pertinência tipo-2 do controlador, utilizando outros métodos de otimização metaheurística como Diferential search(DS) ou outros métodos de otimização. Implementar uma versão deste controlador que utilize algoritmos de redução de tipo alternativos com um menor custo computacional para aplicação em malhas de controle com dinâmica mais rápida. Além de aplicar a mesma estrutura de controle em processos não-lineares.

\section{AGRADECIMENTOS}

Agradeço ao GRASI-UFPI pela disponibilização dos equipamentos.

\section{REFERÊNCIAS}

Almeida, O.d.M. and Coelho, A.A. (2002). A fuzzy logic method for autotuning a pid controller: Siso and mimo systems. In 15th IFAC World Congress on Automatic Control.

Åström, K.J. and Hägglund, T. (1984). Automatic tuning of simple regulators with specifications on phase and amplitude margins. Automatica, 20(5), 645-651.

Åström, K.J. and Hägglund, T. (2001). The future of pid control. Control engineering practice, 9(11), 1163-1175.

Berner, J., Soltesz, K., Hägglund, T., and Aström, K.J. (2018). An experimental comparison of pid autotuners. Control Engineering Practice, 73, 124-133.

Chaiyatham, T. and Ngamroo, I. (2017). Improvement of power system transient stability by pv farm with fuzzy gain scheduling of pid controller. IEEE Systems Journal, 11(3), 1684-1691.

Coimbra, M.D.S., Guaracy, F.H.D., Ferreira, L.H.D.C., and Pugliese, L.F. (2017). Sintonia de controlador fuzzy baseada em algoritmos genÉticos aplicada a um sistema
nÃo linear de levitaÇÃo magnÉtica. XIII Simpósio Brasileiro de Automação Inteligente.

Dai, A., Zhou, X., and Liu, X. (2017). Design and simulation of a genetically optimized fuzzy immune pid controller for a novel grain dryer. IEEE Access, 5, 14981-14990. doi:10.1109/ACCESS.2017.2733760.

Hagras, H. (2007). Type-2 flcs: A new generation of fuzzy controllers. IEEE Computational Intelligence Magazine, $2(1), 30-43$.

Herrera, F., Lozano, M., and Verdegay, J. (1998). Tackling real-coded genetic algorithms: Operators and tools for behavioural analysis. Artificial Intelligence Review, 12(4), 265-319.

Karnik, N.N., Mendel, J.M., and Liang, Q. (1999). Type2 fuzzy logic systems. IEEE transactions on Fuzzy Systems, 7(6), 643-658.

Kruse, R., Borgelt, C., Braune, C., Mostaghim, S., and Steinbrecher, M. (2016). Computational intelligence: a methodological introduction. Springer.

Kwok, D., Tam, P., Li, C., and Wang, P. (1990). Linguistic pid controllers. IFAC Proceedings Volumes, 23(8, Part 4), 205 - 210. 11th IFAC World Congress on Automatic Control, Tallinn, 1990 - Volume 4, Tallinn, Finland.

Mamdani, E.H. (1974). Application of fuzzy algorithms for control of simple dynamic plant. In Proceedings of the institution of electrical engineers, volume 121, 15851588. IET.

Qiao, J., Liu, Z., and Zhang, Y. (2017). Gain scheduling pid control of the quad-rotor helicopter. In 2017 IEEE International Conference on Unmanned Systems (ICUS), 1594-1601. IEEE.

Santana, H.G., Coelho, S.d.S., and Almeida, O.d.M. (2018). Application of multivariable pid controllers in a coupled tank system. In 2018 13th IEEE International Conference on Industry Applications (INDUSCON), 664-671. IEEE.

Tai, K., El-Sayed, A.R., Biglarbegian, M., Gonzalez, C., Castillo, O., and Mahmud, S. (2016). Review of recent type-2 fuzzy controller applications. Algorithms, 9(2), 39.

Takahashi, M. and Kita, H. (2001). A crossover operator using independent component analysis for real-coded genetic algorithms. In Proceedings of the 2001 Congress on Evolutionary Computation (IEEE Cat. No.01TH8546), volume 1, 643-649 vol. 1.

Wu, D. and Nie, M. (2011). Comparison and practical implementation of type-reduction algorithms for type2 fuzzy sets and systems. In 2011 IEEE International Conference on Fuzzy Systems (FUZZ-IEEE 2011), 2131-2138. IEEE.

Wu, D. and Tan, W.W. (2004). A type-2 fuzzy logic controller for the liquid-level process. In 2004 IEEE International Conference on Fuzzy Systems (IEEE Cat. No. 04CH37542), volume 2, 953-958. IEEE.

Zadeh, L. (1965). Fuzzy sets. Information and Control, $8(3), 338-353$.

Zadeh, L.A. (1975). The concept of a linguistic variable and its application to approximate reasoning - i. Information sciences, 8(3), 199-249.

Zhao, Z.Y., Tomizuka, M., and Isaka, S. (1993). Fuzzy gain scheduling of pid controllers. IEEE transactions on systems, man, and cybernetics, 23(5), 1392-1398. 